\title{
Evolution de la maquette de TP d'automatique pour la commande en position d'un papillon motorisé
}

\author{
Didier PEYTAVI ${ }^{(1)}$, Pascal MAUSSION ${ }^{(2)}$ \\ didier.peytavi@toulouse.iufm.fr,pascal.maussion@laplace.univ-tlse.fr \\ (1) IUFM Midi Pyrénées, 118 route de Narbonne, 31078 Toulouse cedex \\ (2) INP-ENSEEIHT, 2 rue Camichel, BP 7122, 30071 Toulouse cedex 7
}

\begin{abstract}
RESUME : Cet article présente une évolution de la maquette papillon d'admission d'air, utilisée comme support de formation à l'automatique. La réalisation d'une commande numérique implantée dans un microcontrôleur PIC et d'un amplificateur à découpage MLI, permet à la maquette de se rapprocher beaucoup de l'application industrielle réelle. Le boîtier de commande dédié à cette manipulation, permet sur cet actionneur embarqué, de tester différentes méthodes d'identification et de commande, principalement basées sur des démarches expérimentales de terrain, d'emploi simple et rapide. La manipulation est utilisée cette année dans la formation des élèves ingénieurs du département de Formation Génie Electrique-Automatique de l'ENSEEIHT (Ecole Supérieure d’Electrotechnique, d’Electronique, d’Informatique, d’Hydraulique et de Télécommunications de Toulouse).
\end{abstract}

Mots clés : automatique, correction numérique, microcontrôleur PIC, injection automobile, système embarqué, travaux pratiques, moteur à courant continu, frottements secs, identification

\section{INTRODUCTION}

Le domaine des systèmes embarqués est une mine d’applications pédagogiques souvent très intéressantes et motivantes pour les étudiants et les enseignants. Il peut concerner le génie électrique, l'électronique, l'informatique industrielle ou l'automatique. C'est dans ce dernier domaine que se situe la didactisation d'une application industrielle automobile proposée dans cet article. D'autres manipulations traitant également de l'automatique, axe numérique [1] ou commande à distance d'une MCC [2] ont déjà été publiées mais pas avec cette orientation commande numérique. Cet article fait suite et complète une manipulation décrite antérieurement dans une précédente publication [3], qui traitait essentiellement de procédures d'identification de terrain, contrairement à [4] qui présentait des méthodes d'identification plus complètes mais de mise en œuvre plus complexe. Néanmoins, un travail complémentaire sur l'identification des frottements secs [5] [6] est mené ici dans cette activité.

La valeur ajoutée de notre travail n'est pas centrée sur l'élaboration de lois de commande comme dans [7]. Nous mettons résolument l'accent sur l'implantation de lois de commande simples (PI, PID) dans un microcontrôleur PIC et donc sur l'identification des contraintes correspondantes (formats, mises en forme, codages, limitations...). Ce domaine des microcontrôleurs n'a que peu été exploité dans des publications pédagogiques [8], [9]. Par ailleurs, notre manipulation intègre un nouvel amplificateur à découpage sur lequel des activités d'apprentissage pourraient être également développées comme dans [10] ou [11].

\section{MISE EN SITUATION : RAPPEL DE LA PRESENTATION DU PAPILLON MOTORISE DANS LES MOTEURS A INJECTION ESSENCE}

Les différents principes sont bien connus : carburation et injection [12], réalisée notamment grâce à l'introduction massive de systèmes de dépollution par catalyse et pour diminuer la consommation. Le moteur est alimenté par un mélange air-carburant réalisé soit avant son introduction dans le cylindre (mélange préalable), soit dans le cylindre (injection directe). La quantité d'air admise est modulée par un volet situé dans la tubulure d'admission, le «papillon », le carburant est dosé par un système d'injection, le tout piloté par un calculateur. L'ouverture du papillon est fonction de la consigne en couple, (appui sur la pédale d'accélérateur) du rapport de boite de vitesses, du régime moteur, de la dépression dans l'admission, de la quantité de carburant injectée.... Le modèle de boîtier papillon motorisé étudié dans nos TP est implanté sur les moteurs essence HPI (Haute Pression d'Injection) d'automobiles de type "familiales ». L'asservissement de la position du papillon utilise un moteur à courant continu avec réducteur $1 / 24$, alimenté par un amplificateur à découpage.

Un appui sur la pédale d'accélérateur, modifie la consigne en couple du moteur et pilote l'injection et l'admission d'air, donc la position du papillon. La demande d'ouverture du papillon est alors une différence de potentiel envoyée par le calculateur embarqué. La position du papillon est fixée par l'action du moteur à courant continu via un amplificateur, luimême commandé par le calculateur. Un potentiomètre double, positionné sur l'axe du papillon après un réducteur, permet au calculateur après filtrage de connaître précisément la position de celui-ci. Un 
ressort de rappel spiralé maintient le papillon en butée basse, (position "Limp Home » = retour maison) en l'absence de toute commande du calculateur d'injection, en cas de panne par exemple.

\section{PRESENTATION DE LA MAQUETTE}

Notre maquette de travaux pratiques est constituée :

- d'un papillon motorisé, d'ordinaire implanté sur une automobile française de moyenne gamme (style Peugeot 307 par exemple), figure 1,

- d'un amplificateur de puissance à modulation de largeur d'impulsion, réalisé à l'aide d'un circuit spécialisé, L6203, figure 2 et figure 3,

- d'une carte de développement et de commande à base de microcontrôleur PIC 16F873, figure 4,

- d'une feuille de calcul dans un tableur.

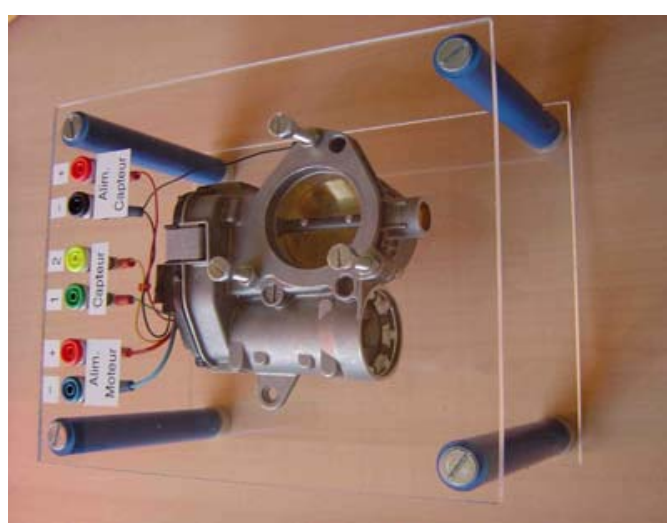

Fig 1 : papillon motorisé
La commande était réalisée l'an dernier en électronique analogique, elle est implantée cette année comme promis sous forme numérique. Elle permet l'implantation des fonctions :

- mesure différentielle et filtrage, pour éviter les bruits et le mode commun,

- génération d'une rampe réglable de consigne de position,

- correction P, PI, PD ou PID,

- $\quad$ filtrage spécifique de l’action dérivée.

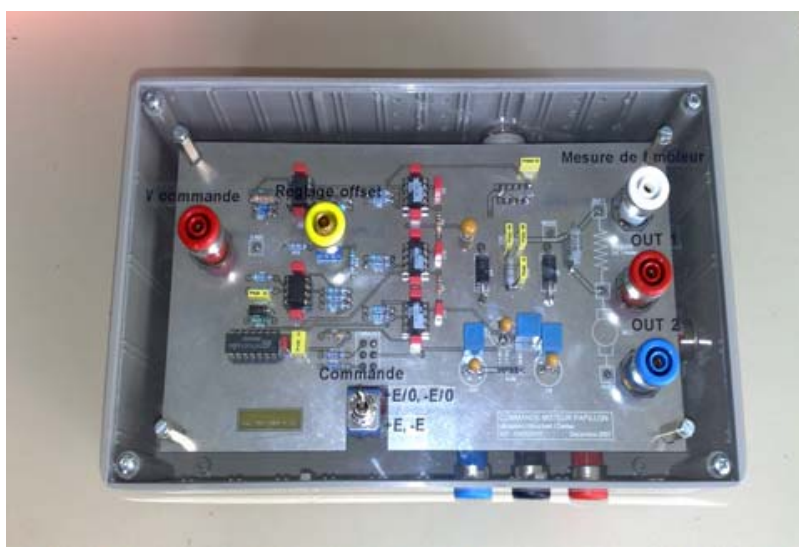

Fig 2 : Amplificateur MLI

Le détail des fonctions de l'amplificateur MLI et leurs implantations sont décrits sur la figure 3. Un amplificateur à découpage est à la base de l'application réelle, aussi avons-nous souhaité remplacer l'amplificateur linéaire de l'an passé, par une structure plus proche de la réalité, basée sur un amplificateur à découpage intégré, le L6203.

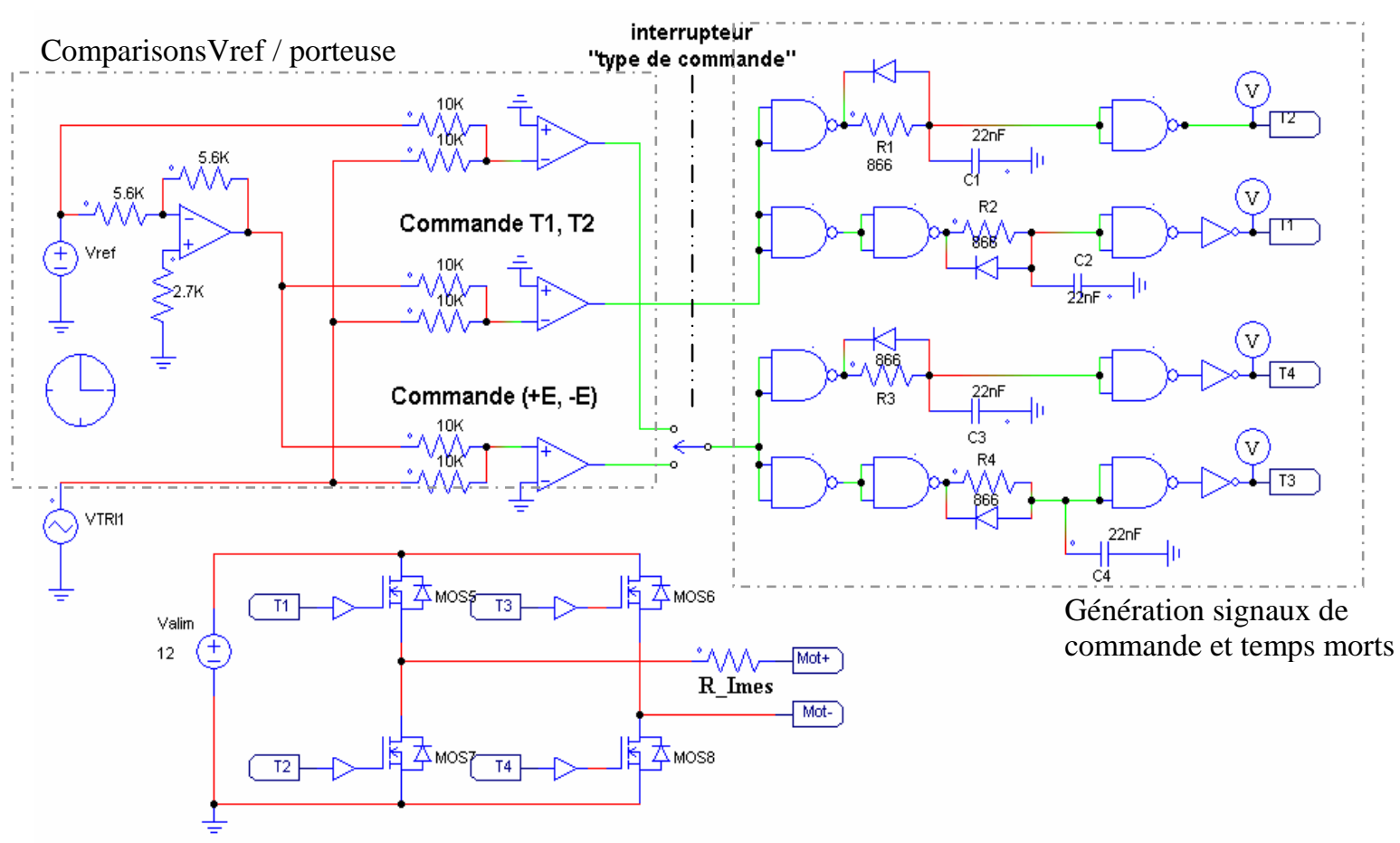

Fig 3 : schéma d'implantation des étages de filtrage et de commande analogique 
La maquette réalisée permet outre sa fonction principale utilisant un microcontrôleur PIC 16F873, de visualiser le courant de sortie dans le moteur. Deux types de commande des interrupteurs sont implantées, elles produisent une MLI 2 niveaux de type $+\mathrm{E} /$-E ou 3 niveaux de type $+\mathrm{E} / 0$ ou $0 /$-E.

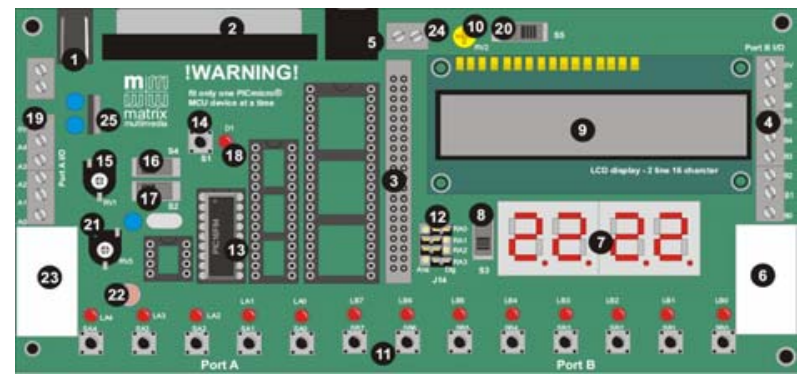

Fig 4 : carte de commande à base de microcontrôleur PIC $16 F 873$

Un inverseur manuel autorise une commutation entre l'un et l'autre des modes et permet la visualisation directe de l'ondulation de courant (amplitude, fréquence) et du bruit de mesure. Les simulations correspondantes ont été réalisées avec PSim [13].

\section{IDENTIFICATION FROTTEMENTS SECS}

DES

Par rapport au TP décrit dans l’article précédent [3], nous ajoutons une activité sur l'identification des frottements secs. En effet, l'identification demandée précédemment aux étudiants, aboutissait à un modèle de Broïda équivalent, valable pour la seule partie linéaire de la courbe de réponse indicielle, cf figure 5.

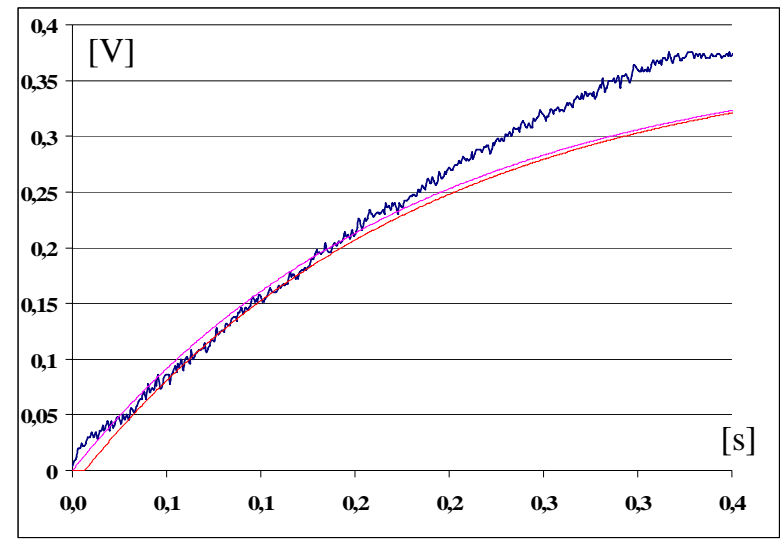

Fig 5 : réponse indicielle expérimentale

Sur la figure5, la non linéarité due aux frottements secs apparaît nettement à la fin du régime transitoire. Il s’agit ici de faire découvrir le phénomène aux étudiants et de rechercher les paramètres d'un modèle dont la forme est fournie de manière pré-déterminée dans l'environnement Matlab-Simulink, cf figure 6

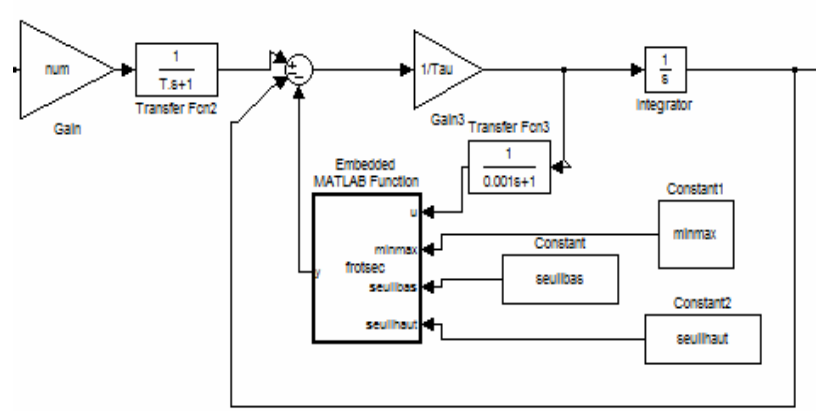

Fig 6: modèle Simulink du frottement sec

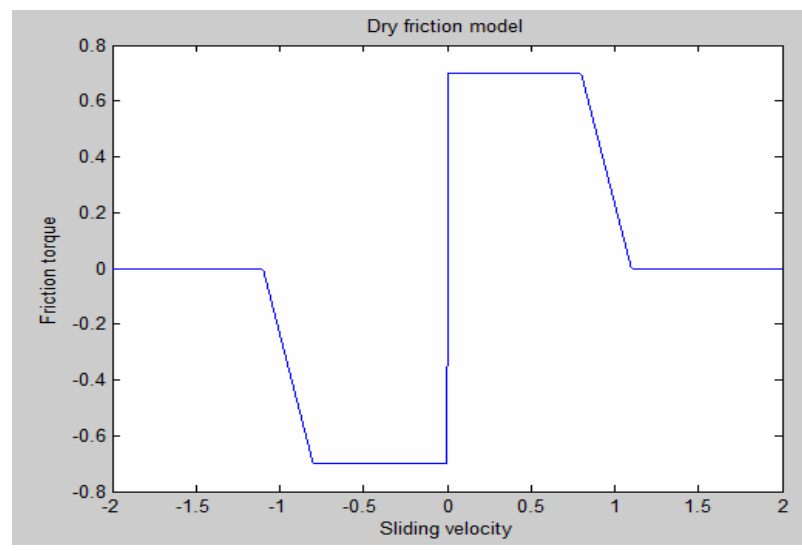

Fig 7 : caractéristique du modèle du frottement sec

L'identification des coefficients du modèle est faite empiriquement, de manière à faire coller la réponse indicielle du modèle avec frottements secs et celle du papillon motorisé, figure 8.

Elle conduit à une courbe figure 7 , caractéristique du frottement sec lui-même. L'insertion de ce modèle de frottements secs dans la boucle d'asservissement conduit à une réponse indicielle plus réaliste, singulièrement différente de la réponse linéaire idéale, comme en atteste la figure 8.

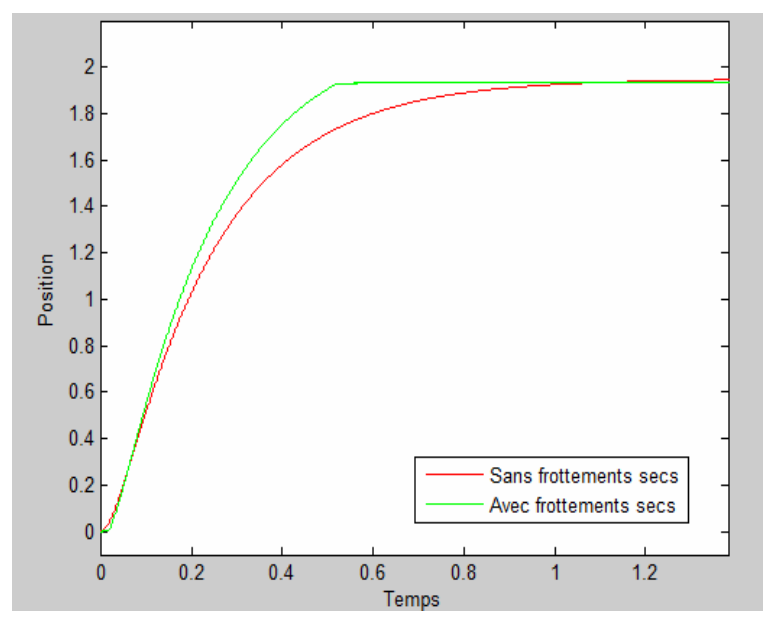

Fig 8: réponses indicielles avec et sans insertion du modèle des frottements secs 
Le correcteur PI, discrétisé par exemple par la méthode des trapèzes, est décrit figure 12. Au rythme de la fréquence d'échantillonnage et dans le sous-programme d'interruption, sont réalisées les opérations de :

- Conversion Analogique Numérique de Vconsigne et Vcapteur ,

- actualisation de Vcna pour la commande du variateur

Le programme principal permet quant à lui:

- le calcul de l'erreur Ncons-Nret,

- le calcul de l'erreur corrigée (régulateur PI),

- la mise au format du signal de commande du variateur avec gestion des saturations.

\subsection{LIMITATIONS TECHNOLOGIQUES LIEES AU CHOIX D'UNE SOLUTION MICRO PROGRAMMEE}

La cadence d’échantillonnage est limitée, par la durée nécessaire au calcul du signal d'erreur et de la régulation PI (calculs réalisés en virgule flottante), mais surtout par les opérations circulaires de conversion analogique numérique du CAN intégré au microcontrôleur (multiplexage analogique des entrées, acquisition et échantillonnage d'une voie à convertir non négligeables en terme de durées).

La carte de développement utilisée (figure 4) étant équipée d'un quartz à 3,2768 Mhz $\left(\mathrm{Fe}_{\max }=10 \mathrm{Khz}\right.$ par voie lors des tests, deux voies à convertir), après compromis nous choisissons de générer une

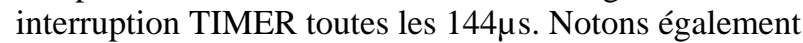
qu'un réseau passif de type $\mathrm{R} 2 \mathrm{R}$ réalise la conversion numérique analogique afin de ne pas rajouter de temps de cycle supplémentaires à la boucle principale de

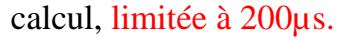

\subsection{RESULTATS EXPERIMENTAUX}

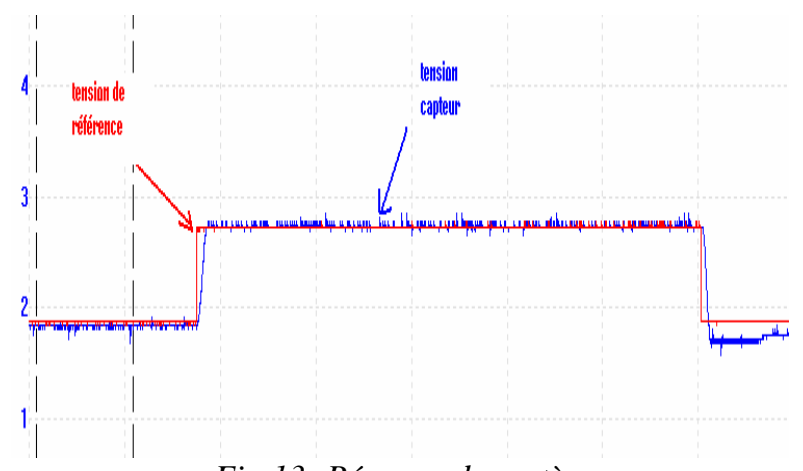

Fig 13: Réponse du système avec un correcteur PI numérique

Un échelon de tension de $1 \mathrm{~V}$ est appliqué en Vconsigne afin de tester la validité du régulateur, cf figure 13 : la réponse suit la référence lors de l'ouverture et de la fermeture du papillon mais avec un léger dépassement pour ce dernier cas de figure. Le temps de réponse $\operatorname{Tr}_{5 \%}$ , ainsi obtenu est de $94 \mathrm{~ms}$.

\section{DESCRIPTION DES TP.}

\subsection{JUSTIFICATION DE L'ORIENTATION DES TP}

Le support présenté dans cet article, va permettre la mise en place d'un ensemble de deux TP. Il est conçu comme une mise en pratique de concepts vus précédemment en cours. Par ailleurs, ce TP de commande numérique complète celui proposé dans l'article précédent [3], qui était centré sur l'identification par analyse de la réponse indicielle et la synthèse de correcteur PI analogiques par des méthodes " industrielles », simples, pratiques et rapides. Ainsi, le même support de formation extrait d'une application industrielle est utilisé dans différentes situations d'apprentissage (cours, TP) et pour différents savoirs ou savoir-faire de l'automatique: depuis l'identification jusqu'à l'implantation de correcteurs discrétisés dans un microcontrôleur. L'idée principale et fondatrice de cet enseignement basé sur des applications industrielles, est de donner une cohérence aux divers apprentissages en les centrant en partie sur un support commun à plusieurs séquences d'enseignement. C'est ce support commun qui donne à nos yeux plus de sens aux apprentissages.

Il s’agit là, modestement mais efficacement, d'une "petite » application industrielle de commande numérique. L'objectif n'y est pas de tester des commandes sophistiquées implantées dans des cartes DSP très performantes mais généralement coûteuses. Il vise au contraire à mettre en évidence les contraintes de l'implantation dans un microcontrôleur: période d'échantillonnage limitée, nécessité de traiter des grandeurs analogiques uniquement positives dans la plage $0-5 \mathrm{~V}$, augmentation du temps de cycle par codage de l'algorithme en virgule flottante... Ces contraintes disparaissent totalement dans un système de développement de commande numérique puissant, mais ô combien plus coûteux et totalement inapproprié dans le contexte de l'électronique embarquée en automobile. A ce titre rappelons que quelques $€$ économisés sur le choix du processeur peuvent générer des sommes importantes vu le nombre d'automobiles fabriquées.

\subsection{OBJECTIFS DES TP}

Les objectifs des TP sont : être capable de

- identifier les fonctions de la carte de commande (hors asservissement), spécifiques au caractère embarqué du dispositif,

- paramétrer le modèle des frottements secs par comparaison des réponses temporelles en boucle ouverte obtenues entre le processus réel et le modèle,

- comprendre le conditionnement analogique des signaux issus des capteurs de position (amplification différentielle et gestion des offsets pour recentrage par rapport à la plage d'entrée du CAN), gestion des facteurs d'échelle pour commander le variateur,

- justifier la valeur de la période d’échantillonnage, intérêt de la mise en œuvre des interruptions liées 
à la fonction Timer (valeur de comparaison atteinte ou overflow) et interactions avec le programme principal de distribution des tâches,

- mettre en œuvre un CAN intégré avec balayage des voies à convertir, vérifier l'adéquation entre résolution de conversion et précision de positionnement du papillon,

- calculer les paramètres d'un réseau correcteur PI ou PID industriel,

- discrétiser le correcteur et l'implanter dans le microcontrôleur,

- estimer les durées de calcul et valider le choix de Te min à programmer,

- vérifier les performances par des mesures,

- expliquer les différences constatées entre prédéterminations et mesures.

\subsection{DIFFICULTES HABITUELLES DES ETUDIANTS ET REMEDIATIONS}

Dans ce type d'apprentissage, les difficultés des élèves ingénieurs, se situent usuellement, plus fréquemment dans la mise en œuvre des lois de commande sur un microcontrôleur que dans l'usage du calcul symbolique ou d'outils de l'automatique. Mais au-delà des «astuces » d'implantation pratique des lois de commande, il nous semble intéressant de viser dans ce type de TP, à faire acquérir un sens des réalités, des limitations technologiques, des contraintes de l'implantation réelle... qui peuvent se perdre à trop fréquenter les environnements de simulation.

\section{CONCLUSION}

Cet article présente l'évolution d'une maquette de TP d'automatique basée sur l'utilisation d'un actionneur électromécanique embarqué en automobile. Elle permet de mettre en évidence les fonctions de commande implantées dans un microcontrôleur et les contraintes associées.

De conception simple, rapide et peu coûteuse, cette maquette ou ses principes peuvent être utilisés au niveau DUT, au niveau L ou au niveau M. L'évolution ultérieure de cette maquette concernera le développement de commandes numériques plus complexes, destinées à augmenter la dynamique et compenser l'effet des frottements secs.

\section{BIBLIOGRAPHIE}

[1] « Axe numérique »,, J.-P. RENAUD, S. GORON et J. GARNIER, CETSIS 2003, Toulouse

[2] «Régulation de vitesse pour MCC : choix d'un contrôle à distance ", TAUVEL A., FLETY E., CETSIS 2005, Nancy

[3] « Maquette de TP d'automatique : identification et commande en position d'un papillon motorisé », P. MAUSSION, CETSIS 2007, Bordeaux
[4] « Montage expérimental didactique pour étudier différentes stratégies d'identification d'un système thermique », L. PEREZ, L. AUTRIQUE, CETSIS 2005, Nancy

[5] « Les frottement : origine physique et modèles », B. BORSOTTO, D.BEAUVOIS, E. DEVAUD et E. GODOY, Technologie et formation, novembre 2006

[6] "Dry friction", wikipedia, http://en.wikipedia.org/wiki/Friction

[7] « Etablissement des lois de commande des machines électriques », G. FELD et E. HOANG, CETSIS 2003, Toulouse

[8] «Actionneurs linéaires : MRVlin et MSlin, un projet pédagogique », L. BAGHLI, A. REZZOUG, CETSIS 2007, Bordeaux

[9] « Pilotage du bus I2C à partir du microcontrôleur HCS12 », Rachid MALTI, Serge BOUTER, Cetsis 2007, Bordeaux

[10] « Mise en oeuvre d'une commande à modulation de largeur d'impulsion pour un hacheur en pont», GAUBERT J.P. , BOSCHE J., CETSIS 2005, Nancy

[11] «Ensemble pédagogique expérimental hacheur onduleur », HUSELSTEIN J.J., MARTIRE T., CETSIS 2005, Nancy

[12] « Moteur à allumage commandé, Composants et stratégie de contrôle », Henri Mazet, BM2970, et BM2971, Techniques de l'ingénieur http://www.techniques-ingenieur.com

[13] http://www.powersys.fr

Nota : Les fichiers de la maquette d'amplificateur MLI sont disponibles sur www.iufmrese.cict.fr/contributions

\section{Remerciements}

Ce TP a été réalisé notamment grâce à :

o Frédéric MOUCHART, étudiant CNAM ingénieur pour la réalisation de l'amplificateur MLI et la complicité de Olivier DAVIAU, Technicien du plateau Automatique,

o EL HARDOUZ Youssef et SAUNIER Julien, étudiants de $3^{\circ}$ année en projet long Automatique et Informatique Industrielle dans la filière GEA de l'ENSEEIHT, pour l'implantation dans le microcontrôleur, durant l’année 2007-2008.

Qu'ils en soient ici remerciés. 